\title{
El cambio organizativo e institucional en las cooperativas de Mondragón: el caso de la cooperativa AMPO
}

\author{
Rafael Altuna \\ Mondragon Unibertsitatea \\ raltuna@mondragon.edu \\ Eguzki Urteaga \\ Universidad del País Vasco \\ eguzki.urteaga@ehu.es
}

Resumen: Este artículo analiza el cambio organizativo e institucional en las cooperativas a través del caso de la cooperativa AMPO, perteneciente al Grupo Mondragón basta el año 2008. Este trabajo pone de manifiesto la aplicación de un nuevo modelo de gestión en dicha cooperativa a partir de 2003, inspirado en la teoría de Koldo Saratxaga. Este último ha propiciado la introducción de cambios en el liderazgo y el estilo de dirección; en la participación y gestión de las personas; en los procesos, estructuras y contenidos del trabajo, $y$, por último, en la cultura organizativa. Estos cambios, junto con una estrategia centrada en el cliente como elemento esencial de todo el proceso, han posibilitado la consecución de buenos resultados cuantitativos.

Palabras clave: cambio organizativo, modelo de gestión, cooperativas, Mondragón.

Organizational and institutional change in the cooperatives of mondragón: the case of the ampo cooperative

Abstract: This article analyzes organizational and institutional change in cooperatives by focusing on the case of the AMPO cooperative, which was a member of the Mondragon Group until 2008. This study reveals how a new management model, inspired by Koldo Saratxaga's theory, was implemented in the cooperative 
in 2003. This has led to changes to leadership and management style, to individual participation and management, to work processes, structures and contents and, finally, to organizational culture. These changes have brought about good quantitative results and a new client-centred strategy that has been the essential to the whole process.

Keywords: organizational change, management model, cooperatives, Mondragon. 


\section{Introducción}

La cooperativa AMPO, perteneciente al Grupo Mondragón hasta el año 2008, inició, a partir del año 2003, un proceso de cambio organizativo e institucional en la búsqueda de un proyecto socioempresarial propio cimentado en la voluntad y capacidad de las personas que la integran.

El análisis que se expone a continuación tiene como base la teoría neoinstitucional (March y Olsen, 1976; Meyer y Rowan, 1977; Power y DiMaggio, 1991; Scott, 1995). Si bien dentro de esta perspectiva coexisten diversas posturas, todas asumen la autonomía de los actores, pero entramada en redes y relaciones intermedias entre el actor y las macroestructuras (Granovetter, 1985; Putnam, 1993; Urteaga, 2013). A lo largo de esta exposición, concebimos la cooperativa AMPO como una institución social, entendida como un conjunto de «normas culturales que proporcionan recursos y valores compartidos a las entidades y a las actividades particulares integrándolas en grandes proyectos (...). Sistemas simbólicos, formas de ordenar la realidad y de proporcionar una experiencia en el pasado y en el tiempo» (Scott, 1994: 58).

\section{Antecedentes y contextualización}

La cooperativa AMPO está situada en el municipio de Idiazabal, perteneciente a la comarca del Goierri ${ }^{1}$ (Gipuzkoa, País Vasco), de larga tradición siderometalúrgica.

La cooperativa AMPO, tal y como está constituida actualmente, es el resultado de la fusión de las empresas Ampo (Aceros Moldeados y Promoción Obrera) y Poyam (Promoción Obrera y Automatización Maquinaria), creadas en los años 1964 y 1962 respectivamente, y fusionadas en el año 1971. En la actualidad alberga tres divisiones en su seno: fundición, válvulas e ingeniería. Las cooperativas originarias fueron creadas por jóvenes trabajadores de la comarca del Goierri deseosos de cambiar el sistema socioeconómico establecido. Así, decidieron crear una nueva empresa en el ámbito de la fundición, dado que era lo que conocían. La opción cooperativa fue elegida en gran medida por la necesidad de recursos financieros, ya que sus tres iniciadores carecían del capital necesario para afrontar semejante iniciativa empresarial. No obstante, en su ánimo también estaba mejorar las condiciones sociolaborales de los trabajadores (Barandiaran, 2000).

1 La comarca del Goierri, situada la parte suroriental de Gipuzkoa, limitando con Álava y Navarra, está constituida por los siguientes municipios: Altzaga, Arama, Ataun, Beasain, Gabiria, Gaintza, Idiazabal, Itsasondo, Lazkao, Legorreta, Mutiloa, Olaberria, Ordizia, Ormaiztegi, Segura, Zaldibia, Zegama y Zerain. En la actualidad su población ronda los 40000 habitantes y su extensión es de en torno a $325 \mathrm{Km} 2$. Olaberria, es el municipio del País Vasco con el Producto Interior Bruto (PIB) per cápita más elevado de la CAPV en el año 2011, según datos publicados por Eustat (Instituto Vasco de Estadística). 
Históricamente, la actividad de AMPO se ha basado en trabajos muy manuales, en no pocos casos ejercidos en condiciones duras y exigentes. En la actualidad, AMPO ocupa posiciones de liderazgo a nivel mundial en segmentos especializados como el de válvulas para servicios criogénicos de tratamiento o el de transporte, manipulación y almacenamiento de gases licuados. Su permanente relación con los clientes le ha llevado a la creación de la división de ingeniería, en parte con el fin de satisfacer la demanda de integración de la ingeniería del proceso por parte del cliente y, en parte, por el conocimiento que aportan a nivel de ingeniería de piping.

En el año 2003, inmersa en una importante crisis económica, la cooperativa $A M P O$ comenzó a poner en práctica su nuevo modelo de gestión. En aquel momento, AMPO formaba parte, junto con las cooperativas Irizar y Urola, de la Agrupación Goikide, perteneciente al Grupo Mondragón. Estas cooperativas compartían, además de la proximidad física por estar situadas en el mismo valle, su inquietud por seguir un mismo modelo de gestión empresarial. Entre ellas se fueron generando múltiples complicidades, en la medida en que compartían las mismas prácticas organizativas. Este hecho generó, a medio plazo, el surgimiento de tensiones en el seno de Mondragón Corporación Cooperativa (MCC) y, fruto de esas tensiones y desavenencias, en el año 2008, tanto Irizar como AMPO se desvincularon de MCC. La prensa se hizo eco de este hecho, lo que dio lugar a una amplia controversia social y mediática respecto a las verdaderas razones que habían provocado una decisión sin precedentes en el cooperativismo de Mondragón. ${ }^{2}$

\section{El modelo de gestión para el cambio organizativo e institucional}

El modelo de gestión que se implanta en AMPO a partir del año 2003 es el inicio de un proceso de cambio organizativo e institucional encaminado al logro de resultados económicos que aseguren el futuro de la cooperativa. En este proceso, Koldo Saratxaga jugó un rol decisivo como ideólogo e impulsor del nuevo modelo que se iba a implantar.

Koldo Saratxaga, directivo empresarial y gurú del management, ${ }^{3}$ asesoró a la cooperativa AMPO a partir del año 2003 en la implantación del modelo de

2 «La brecha abierta se ha cimentado en un choque de culturas. Varios cooperativistas han asegurado [...] que una causa del desencuentro es que Irizar y Ampo, pertenecientes junto con Urola al grupo Goikide, han trabajado con modelos más ágiles de gestión que los aplicados desde la cúpula de Mondragón y que les ha permitido tomar decisiones estratégicas con rapidez para apuntalar su crecimiento». (Cinco Días, 20-5-2008).

3 Koldo Saratxaga (Sopuerta, Bizkaia, 1947) implantó su modelo basado en las personas en la cooperativa Irizar (1991-2004), donde obtuvo resultados espectaculares en todos los ámbitos. En el año 2000 logró el Premio 
gestión que tan buenos resultados había dado en la cooperativa Irizar, y que había dado lugar a un isomorfismo institucional de carácter mimético dentro del grupo cooperativo Goikide, perteneciente a MCC.

La propuesta de Saratxaga gira en torno a dos elementos fundamentales: por una parte, el cambio cultural de la institución y, por otra parte, la aplicación de la reingeniería a todos los procesos organizativos. Todo ello queda articulado en el modelo de gestión.

Desde un punto de vista analítico, son tres los aspectos más relevantes del modelo de gestión de la cooperativa AMPO: la dimensión simbólico-cultural, la dimensión técnico-operativa y la dimensión estratégica.

\subsection{Dimensión simbólico-cultural : el cambio institucional a través del liderazgo transformacional inculturizado}

En el año 2003, AMPO define su misión en los siguientes términos:

Ampo S. Coop. es un proyecto empresarial basado en las personas que trabajando en equipo y mediante la continua satisfacción de las personas, clientes, colaboradores externos y nuestro entorno (sociedad y medio ambiente), nos permita obtener beneficios que hagan posible un crecimiento generador de riqueza y nuevos empleos en un marco cooperativo, de comunicación, libertad y responsabilidad.

En la misión, la cooperativa queda definida como «proyecto empresarial basado en las personas». El cooperativismo como concepto tiene relevancia en la medida en que proporciona un marco institucional adecuado para los objetivos que se pretende lograr. Por tanto, tiene una utilidad pragmática e instrumental.

El acento de la misión pivota en torno a dos elementos: las personas y la obtención de beneficios. La obtención de beneficios como objetivo central de la cooperativa queda expresada sin ambages en la misión de AMPO. Para lograrlo, las personas han de constituirse en las protagonistas. Ello requiere del liderazgo transformacional que provoque un cambio en las personas y en las organizaciones a través de las modificaciones institucionales (normativas, cognitivas y reguladoras) precisas.

Europeo a la Excelencia Empresarial. Debido a ello, su relevancia traspasó el ámbito meramente empresarial y se constituyó como un referente social y mediático. En el año 1997 recibe el Premio Emprendedor del Año en España, concedido por Ernst \& Young, La Caixa y Cinco Días y, en el año 2001, el Premio Mejor Empresario del País Vasco. 


\subsubsection{Liderazgo transformacional}

Las personas que integran AMPO son el eje principal para el logro de los objetivos propuestos. Alinear a las personas con los objetivos de acuerdo con los pensamientos estratégicos definidos es la tarea de un líder transformacional. ${ }^{4} \mathrm{La}$ función del liderazgo estriba, básicamente, en ofrecer a la organización una clara visión de futuro, comunicarla, involucrar a las personas y comprometerse con los esfuerzos que exige su implantación, predicando con el ejemplo. En AMPO, este liderazgo lo encarnan, junto con Koldo Saratxaga, el coordinador general y el presidente del Consejo Rector. Los tres van a ser figuras de gran relevancia en todo el proceso. Saratxaga, por su carisma y prestigio logrado en Irizar; el coordinador general, por su amplia experiencia en la gestión empresarial, y el presidente, por su carácter joven y dinámico. Todos ellos sabrán ganarse la confianza de los trabajadores.

A los dos meses de la incorporación de Saratxaga a AMPO como asesor, este nombró coordinador general a una persona con gran experiencia en gestión, dado que era uno de los directores de negocio de la cooperativa Orkli (perteneciente a MCC y situada en la comarca del Goierri). También contaba con gran prestigio a nivel social, dado que desempeñaba un cargo de responsabilidad política en un ayuntamiento de la comarca del Goierri y era poseedor de una visión profundamente ética de la actividad empresarial, sustentada en valores cristianos. Además, se identificaba plenamente con la figura de Saratxaga y con el modelo de gestión que este había impulsado en Irizar.

A estas dos personas, líderes del proceso de transformación, hay que sumar al presidente del Consejo Rector. Desde el año 2001, el puesto lo ocupaba una persona joven, dinámica y dispuesta a aportar su esfuerzo generoso al proceso de cambio. Esta persona, con un conocimiento suficiente de la cooperativa, no arrastraba, sin embargo, una memoria histórica que le dificultara asimilar e impulsar el cambio, algo imprescindible para la creación de un futuro más prometedor para la cooperativa. $\mathrm{Al}$ igual que el coordinador general, también tenía responsabilidades políticas en un ayuntamiento de la comarca del Goierri. Ambos compartían una misma ideología nacionalista vasca.

\footnotetext{
4 El liderazgo transformacional es un estilo de liderazgo que se define como un liderazgo que crea un cambio valioso y positivo en los seguidores. Un líder transformacional se centra en «transformar» a otros para que se ayuden mutuamente, para que se preocupen por los demás, para que estén alentandos y armoniosos, y para que miren la organización como un todo. En este mandato, el líder aumenta la motivación, la moral y el rendimiento de su grupo de seguidores. James MacGregor Burns (1978) y Bernard M. Bass (1985) son quienes elaboraron el concepto.
} 
Estas tres personas van a liderar, desde un punto de vista técnico, de gestión e institucional, el proceso de transformación de AMPO, siguiendo la estela de la cooperativa Irizar pero con sus peculiaridades propias. Lo que tratan de generar dentro de AMPO es un cambio cognitivo, regulador y normativo. Todos ellos se retroalimentan, de modo que los cambios normativos reforzarán la credibilidad del modelo y los aspectos reguladores,, generarán una mayor confianza y posibilitarán los cambios en las actitudes y comportamientos de los trabajadores de AMPO.

\subsubsection{Cambios normativos, reguladores y cognitivos}

El logro de los objetivos propuestos hace necesaria la transformación institucional de la cooperativa en sus aspectos cognitivos (culturales), reguladores (comportamientos y valores) y normativos (normas y códigos de conducta). El punto de partida de la transformación gira en torno a las propuestas de cambio cultural realizadas por Saratxaga (2007) y que son, de manera resumida:

- Proyecto basado en las personas. «Debemos pasar de un estilo «yo-ello», que es una forma de relacionarse con algo cuyo valor es instrumental -y de aportación material en todo caso- a un estilo «yo-tú», donde son los valores intrínsecos de las personas los que cuentan».

- Equipos autogestionados. «El sistema jerárquico de «ordeno y controlo» clasifica a las personas de la organización: obreros y empleados; directores, jefes, encargados, operarios; mano de obra directa o indirecta. En función de ello, se crea la dinámica operativa y repetitiva en la que unos pocos piensan por la mayoría. Primer grave error. Las capacidades, la inteligencia y los valores de las personas no tienen mucho que ver, en principio, con su titulación, ni con su posición [...] en la estructura organizativa. Las personas somos portadoras de valores y capacidades: sin embargo, el modelo centenario imperante nos clasifica y decide quién piensa y ordena y quién controla y realiza. Lo malo es que en todos los casos, independientemente de la clasificación jerárquica, el sistema nos lleva a la repetición, a la tarea, a la falta de creatividad».

- Comunicación, libertad y responsabilidad. «Se hace todo mucho más complicado cuando no hay comunicación sincera, cuando la verdad de cada cual no es lo habitual en la comunicación».

- Ofrecer oportunidades, no controlar y dejar cometer errores. «iDadles espacios, dejadles soñar!». 
- Clientes-proveedores integrados. «Necesitamos ampliar nuestro campo de acción, necesitamos personas con más experiencias y conocimientos internos, con una relación mucho más transversal con el total de nuestra organización, con una visión de conjunto del proyecto global, con un sentimiento de pertenencia al conjunto y no a un departamento aislado, y que sientan la necesidad de la ayuda de las demás personas para lograr la fidelidad del cliente como objetivo final».

- Experiencia compartida. «Para incrementar el conocimiento es necesario compartir información y experiencia. Hay muchos ejecutivos que creen que la información y el conocimiento del entorno en el que se desarrolla la actividad se deben suministrar en función del nivel de formación y responsabilidad de la persona en cuestión. Estos ejecutivos, con su cultura del pasado, no llegan a entender que muy pocas personas podrán realizar un trabajo excelente y novedoso si tienen un conocimiento reducido o parcial de las condiciones que atañen a su labor».

- Perspectiva de futuro. «Si visualizamos qué futuro queremos para nuestra organización y este es compartido, las posibilidades de encontrarnos con él aumentan considerablemente».

- Continua sensación de siembra. "Si comparto la siembra, es decir si hemos trabajado y preparado juntos la tierra para la campaña, si hemos analizado qué, cómo y cuándo sembrar, si hemos previsto qué queremos o podemos obtener de esa semilla, si, entre todos, hemos hecho un seguimiento cuidadoso de la evolución, está claro que será más fácil analizar y sacar conclusiones de qué ha pasado y aprender para mejorar en el año siguiente».

- Transparencia-confianza-generosidad. «Las personas son responsables cuando se confía en ellas».

- Orgullo de pertenencia. «El orgullo de pertenencia aumenta la motivación y el interés por un logro superior».

- Evolución física, mental y emocional. "¿Tenemos los líderes o los gestores adecuados y capaces de realizar el cambio? ¿Son capaces de poner a soñar a esas personas que, aparte de unas manos y unos músculos, tienen un cerebro, un corazón y un estómago?».

- Un proyecto común = un éxito compartido. «Un nuevo estilo de relaciones nos lleva a crear un proyecto común y a disfrutar de un éxito compartido. A todas las personas nos gusta el éxito.» 
A partir de estos planteamientos que establecen el marco de actuación, y de cara a la puesta en práctica del nuevo modelo de gestión, se hace necesario provocar el cambio cognitivo y normativo. Para ello, se aprueban los nuevos valores de la organización.

\section{Cuadro 1. Valores de la cooperativa AMPO}

- Hacer de la satisfacción del cliente nuestra mayor prioridad, ya que ello garantiza el futuro de nuestra empresa.

- Conseguir el mayor grado de competencia profesional en todo momento.

- Promover y gestionar cambios, tomando iniciativas y riesgos.

- Trabajar como parte de un equipo contribuyendo con ideas.

- Confiar en otros y a su vez ser merecedores de confianza.

- Comunicar e informar abiertamente. Obtener y compartir conocimiento.

- Respetar a clientes, proveedores, compañeros, la comunidad local y el medio ambiente.

- Aceptar responsabilidades y ser responsables de nuestras acciones.

- Buscar la excelencia. Hacerlo bien a la primera. Cero defectos.

- Hacer de la calidad, el servicio, el coste, la innovación, la seguridad y la salud en el trabajo y las experiencias compartidas las claves de nuestra actividad.

Fuente: AMPO.

Los valores de la cooperativa están encaminados al logro de los objetivos de la organización. Para ello, su estilo de funcionamiento está centrado en los clientes y en los miembros de la organización. El cambio cultural se ha de traducir en un cambio en sus actitudes y comportamientos: trabajo en equipo, iniciativa, riesgo, confianza, comunicación abierta, responsabilidad y excelencia.

Los cambios a nivel cognitivo, siempre difíciles, han de ser acompañados por cambios reguladores y normativos. Entre ellos, cabe destacar el cambio en el sistema retributivo, con el cual se establecen cuatro niveles salariales básicos. De los 450 trabajadores de AMPO, en torno a 350 pasaron a tener el mismo salario base, el denominado «salario base profesional». Anteriormente, había en torno a 100 nóminas con diferente base salarial, lo que dificultaba enormemente realizar un trabajo en equipo en términos de colaboración, solidaridad, rigor y transparencia. A partir de ahí, se establecen tres niveles salariales más: el de formación universitaria (ingeniería); el de los coordinadores de equipos completos y, por último, el nivel gerencial. Con este sistema, AMPO recupera un elemento muy relevante desde un punto de vista de los principios cooperativos y desde la credibilidad como institución cooperativa, al poner en valor el principio de solida- 
ridad retributiva, asumiendo y poniendo en práctica la equidad como elemento de identidad.

Desde la coordinación general se lanzan mensajes y se fomentan comportamientos tendentes a la eliminación de privilegios y a la reducción del apego al poder derivado del puesto ocupado, subrayando la máxima de que el hecho de mandar o de liderar mejor consiste básicamente en servir a las personas, estar disponible y ayudarlas a lograr sus objetivos. Se estimula y se potencia el trabajo en equipo, eliminando jerarquías y figuras anteriormente relevantes como encargados, etc. Se potencia la rotación de puestos para evitar el apego a los puestos y a los privilegios que conllevan.

Un segundo aspecto de la gestión de las personas ha sido el cambio en el tratamiento que se ha dado a los trabajadores eventuales. La política seguida a lo largo de estos años ha sido la de posibilitar la incorporación de estos trabajadores como socios de la cooperativa. A partir del año 2008, y a diferencia de otras cooperativas, en AMPO han mantenido el puesto de trabajo a los eventuales aun a costa de reducir los niveles salariales del conjunto de trabajadores. Este hecho es una muestra del nivel de solidaridad alcanzado, lo cual ha incidido positivamente en el compromiso y la implicación de todos en el proyecto cooperativo.

Hay que señalar, por último, que estos valores y esta nueva cultura organizacional de AMPO han logrado conectar con el sustrato cultural del entorno en el que se inserta la cooperativa. El liderazgo transformador de AMPO elabora un discurso que conecta con el universo cultural de sus miembros y establece unas normas en coherencia con él. En la medida en que estos elementos están integrados de manera coherente, es más factible que la cooperativa vaya logrando los objetivos en sus diferentes vertientes. $Y$ es en este proceso donde radica gran parte del éxito logrado por AMPO.

\subsection{Dimensión técnico-operativa: reingeniería de procesos a través de equipos de trabajo}

La organización interna de AMPO ha sufrido una notable transformación a partir del año 2003. Ha sido implantada una organización de carácter horizontal orientada al cliente, donde se han suprimido figuras como encargados o directores, y se han sustituido por equipos con diferentes cometidos. El modelo organizativo ha roto con las barreras jerárquicas y departamentales. La base del modelo son las personas organizadas en equipos, con múltiples relaciones entre ellas.

Desde un punto de vista técnico, utiliza las herramientas y los procedimientos aportados por la reingeniería. El concepto de reingeniería asumido por AMPO 
está basado en la conceptualización realizada por Hammer y Champy (1994). ${ }^{5}$ En el proceso de implementación contaron con el apoyo de personas de la cooperativa Irizar, que tenía un amplio recorrido en este ámbito. La cooperativa Irizar ha sido, por lo tanto, un referente y un apoyo fundamental en este proceso, prácticamente el único con que han contado, dado que apenas existen consultoras o asesores en el mercado con conocimientos suficientes en estos aspectos. Por otro lado, estos procesos de transformación organizacional tienen un alto nivel de experimentación, dado que el componente esencial no son las técnicas y herramientas que se han de implementar, aun siendo imprescindibles, sino las personas que las aplican y las lideran. Por tanto, en cada caso y circunstancia, los procedimientos pueden modificarse para que se adapten mejor a cada organización. Eso fue lo que hizo AMPO, pero siempre recorriendo la senda marcada por su predecesora.

En el ámbito de fabricación, la organización cuenta con diferentes Equipos de Línea-Cliente (ELC). En la división de fundición cuentan con cuatro ELP: Alfaset, Oskola, Replicast y Tubos Centrifugados. En la división de válvulas cuentan con siete líneas, en función del tamaño de las piezas.

Junto a ellos, se crearon los Equipos de Satisfacción del Cliente (ESC) en sustitución del departamento comercial. Estos equipos relacionan a la empresa con los clientes. Cuentan con un líder y con dos o tres personas más, organizados en función del área geográfica a la que están enfocados.

En el organigrama están integrados los proveedores y los clientes. Por último, también quedan integrados diferentes servicios: servicios tecnológicos (diseño, planificación, etc.), por un lado, y servicios generales (personal, administración, compras, servicio médico, mantenimiento, etc.), por otro lado. Se incluyen además los servicios subcontratados.

Veamos, a continuación, con mayor detenimiento, los diferentes equipos que constituyen la cooperativa:

\section{a) Equipos de satisfacción del cliente (ESC)}

Los Equipos de Satisfacción del Cliente están formados por tres personas que tienen experiencias y conocimientos diferentes, de manera que sean lo más variados y multidisciplinares posible. Tienen un líder, un reto y un plazo de cumpli-

\footnotetext{
5 La reingeniería o BPR (Business Process Reingeniering) surge en los Estados Unidos a comienzos de los años noventa del pasado siglo como consecuencia de dos hechos fundamentales: el avance de la investigación en sistemas de información y el desarrollo del movimiento de la Calidad Total. Así, la reingeniería puede interpretarse como una ramificación del movimiento Total Quality Management cuyo soporte fundamental lo constituyen tres ideas: la gestión basada en procesos, la utilización de tecnologías de información aplicadas a estos y, finalmente, la implantación radical del cambio. De acuerdo con Hammer y Champy (1994: 42), la reingeniería se define como «la revisión fundamental y el rediseño radical de procesos para alcanzar mejoras espectaculares en medidas críticas y contemporáneas de rendimiento, tales como costes, calidad, servicio y rapidez».
} 
miento. Están presentes en el mercado y conocen de cerca a los clientes. Toman parte en la concepción de los productos, en su proceso y en la dinámica interna. Reciben información de la oferta y demanda de productos y se la transmiten en tiempo real, a través del sistema informático, a los Equipos de Línea-Cliente.

\section{b) Equipos de Linea-Cliente (E.L.C.)}

Los Equipos de Línea-Cliente agrupan a los trabajadores de fabricación y las líneas de producción. En función del producto y del proceso establecido, están formados por entre tres y seis personas. Tienen funciones y responsabilidades claramente definidas, para responder en cantidad, plazo y calidad a los clientes internos y externos. Debido a ello, se pueden hacer las mejoras e innovaciones necesarias en torno a la calidad, al servicio, al coste y a la innovación. Estos equipos nombran a sus líderes, que no tienen por qué ser siempre los mismos.

\section{c) Equipos de relación con proveedores}

El objetivo de estos equipos es proveer a la cooperativa con los materiales y componentes necesarios en el momento preciso, con la cantidad y la calidad adecuadas. Además, a través de la integración de proveedores y clientes, han de conocer sus demandas y compartir los conocimientos adquiridos.

\section{d) Equipos de reingeniería de compras}

Estos equipos han de reinventar y rediseñar la manera de realizar las compras. Los miembros que integran estos equipos no son miembros del área de compras y entre ellos no hay relación funcional ni jerárquica. El objetivo es reinventar y no actuar de acuerdo con las experiencias del pasado ni estar sometidos a su influencia.

\section{e) Servicios tecnológicos}

Estos equipos tienen la función de facilitadores; esto es, sirven a los procesos internos pero siempre en conexión con el cliente externo. Ello les exige tener un conocimiento preciso de los procesos clave y del mercado. En ellos se sitúan los servicios de diseño, planificación, seguridad, calidad, etc.

\section{f) Servicios generales}

En los servicios generales se sitúan aquellos que prestan servicios a todos los trabajadores: servicio médico, administración, informática, etc. 


\section{g) Equipos de pilotaje}

La razón de ser de estos equipos consiste en hacer un seguimiento de los objetivos estratégicos del proyecto y asegurarse de que se están realizando las acciones necesarias en los diferentes ámbitos de la organización para el correcto cumplimiento de estos objetivos. En el equipo de pilotaje están presentes los líderes de las diferentes áreas.

El equipo de pilotaje ha sufrido modificaciones desde el año 2003, cuando comenzó su actividad. En un principio, estaba constituido por un solo equipo en el que participaban los coordinadores de diferentes procesos (finanzas, calidad, servicios tecnológicos, compras, mantenimiento, planificación, etc.), junto con miembros del ESC y, en determinados momentos críticos, líderes de determinados equipos y procesos. Los miembros del equipo de pilotaje no eran estables ni totalmente permanentes en el tiempo, sino que se integraban o dejaban de hacerlo en función de las prioridades y necesidades del momento. Se reunían una vez al mes y, en dicha reunión, revisaban el denominado Plan de Ideas y Objetivos (PIO). Esto es, se establecía la estrategia de la empresa a largo plazo (aspectos cualitativos) y los objetivos concretos del mes correspondiente (aspectos cuantitativos).

Posteriormente, para hacer un seguimiento de los objetivos establecidos, se crearon dos grupos de planificación formados por los distintos líderes de los ELC; uno de ellos en representación de los equipos Oskola, Alfaset y Replicast y el otro para el equipo de centrifugado. Estos grupos se reunían los viernes con un objetivo definido: hacer un seguimiento del grado de cumplimiento de los objetivos de producción y cumplir con las previsiones, para satisfacer las demandas de los clientes. En esta tarea también los ESC jugaron un importante papel.

A partir del año 2009, una vez detectadas las carencias en el funcionamiento de los equipos de pilotaje que dificultaban el logro de los objetivos estratégicos, se pusieron en funcionamiento los doce Equipos Operativos de Pilotaje: cinco en la división de fundición y siete en la división de válvulas. En ellos participan 144 trabajadores (un $30 \%$ de la plantilla). Por tanto, podemos decir que el $95 \%$ de la plantilla tiene representación directa en estos equipos.

Miembros de los ESC, de los ELP y de los servicios tecnológicos forman parte de los Equipos Operativos de Pilotaje. Veamos qué es lo que aporta cada uno de ellos a este equipo.

Los ESC nombran un representante o líder para cada uno de los Equipos Operativos de Pilotaje. Su cometido es transmitir la información que ha sido tratada por el ESC conjunto; esto es, transmitir, de cara a los distintos mercados que 
se trabajan, las oportunidades que se detectan y las previsiones que establecen. Lo tratado en los Equipos Operativos de pilotaje también se transmitirá a los ESC.

El planteamiento para los ELC es similar. Todas las personas que toman parte en las actividades productivas comunes a diferentes ELP forman un equipo, $y$ tienen su representación en alguno de los Equipos Operativos de Pilotaje.

En relación con los servicios tecnológicos, estos engloban los servicios de seguridad, calidad, diseño y planificación. Estos servicios también tienen su presencia en la reunión semanal de los viernes de los equipos Operativos de Pilotaje.

Hay quen señalar, por último, que cada uno de estos equipos elige a su líder para que los represente en cada uno de los Equipos Operativos de Pilotaje y entre todos ellos elijen al líder de los distintos Equipos Operativos de Pilotaje.

Los objetivos de los Equipos Operativos de Pilotaje son los siguientes:

- Tasa de servicio. Cada Equipo Operativo de Pilotaje establece su tasa de servicio, de modo que su responsabilidad consiste en alcanzar los objetivos propuestos y en impulsar las acciones necesarias para lograr el objetivo evitando retrasos.

- Calidad. Los objetivos de calidad se establecen en los equipos. Es responsabilidad del Equipo Operativo de Pilotaje impulsar acciones para reducir las faltas de calidad, realizando las auditorías necesarias y mediante el autocontrol.

- Seguridad. Los objetivos en torno a la seguridad se encuentran también entre los objetivos de los Equipos Operativos de Pilotaje.

- Rentabilidad. Cada equipo tiene su cuenta particular de resultados, de modo que la rentabilidad alcanzada se analiza en función de los equipos.

- Mejora continua, orden y limpieza. Los Equipos Operativos de Pilotaje tienen responsabilidades sobre temas relacionados con el orden y la limpieza, los proyectos de mejora, etc.

\subsection{Dimensión estratégica pensamientos estratégicos orientados al cliente}

Todos los años se hacen un par de jornadas de reflexión conjunta con la participación de unas 40-50 personas pertenecientes a los distintos equipos de AMPO (ESC, ELC, planificación, diseño, etc.). Además, se celebran dos asambleas anuales (en mayo y en diciembre). En la asamblea de diciembre, se ratifican y acuerdan entre todos los miembros de AMPO las «ideas y objetivos» (o «plan de gestión consensuado en asamblea»), con los retos más importantes que se marcan para 
el año siguiente. En la asamblea de mayo se lleva a cabo un seguimiento de estos objetivos.

Posteriormente, se hace un seguimiento de lo acordado en las reuniones de pilotaje mensual (25-30 personas), así como en las reuniones de planificación semanales, en las que llegan a intervenir de forma directa más de 120 personas cada semana.

\section{Valoración del modelo de gestión en relación con el cambio organizativo e institucional}

Desde el momento de su implantación, el modelo de gestión de la cooperativa AMPO tiene una clara orientación hacia el cambio. Desde su inicio, y como consecuencia de la situación de crisis que padecía, la organización en su conjunto, bajo el liderazgo del Consejo Rector, percibe como necesaria y urgente la transformación de la organización en los aspectos básicos de su funcionamiento. Este cambio se aborda de manera explícita y consciente por parte de los miembros de la organización, que asumen el reto del cambio como la única alternativa posible ante una situación económica que pone en riesgo su viabilidad. Este cambio está enfocado al logro de resultados económicos de manera prolongada en el tiempo y de forma autocentrada; esto es, a partir de la movilización de los recursos, sobre todo humanos y organizativos, pero también tecnológicos y financieros, con los que cuenta la cooperativa.

Analizaremos los cambios que se han producido en la cooperativa como consecuencia de la aplicación de este modelo de gestión, en siete variables organizativas: liderazgo y estilo de dirección, organización basada en procesos, contenido del trabajo, estructura organizativa, participación de los trabajadores, cultura y gestión de las personas.

\subsection{Liderazgo y estilo de dirección}

Desde el inicio del proceso de implantación del nuevo modelo de gestión, se han producido cambios sustanciales en el estilo de dirección en AMPO. Se trata de un estilo de dirección que promueve la relación directa entre todos los miembros de la organización, desde la cercanía, la comunicación y la transparencia. Se producen dos Asambleas Generales anuales entre todos los miembros de la cooperativa, además de las reuniones mensuales de seguimiento y las reuniones semanales de planificación. El conjunto de trabajadores está permanentemente informado de manera precisa, gracias al uso de un lenguaje inteligible, de la situa- 
ción de la empresa, de los pedidos, clientes, márgenes de beneficios, situación de la competencia, etc.

El modelo se ha ganado la credibilidad del conjunto de trabajadores (socios y no socios), en la medida en que se han implantado políticas de gestión del personal basadas en la equidad. Prueba de ello es el sistema retributivo basado en cuatro niveles salariales.

Junto a ello, cabe destacar el trato dado a los trabajadores por cuenta ajena con contrato temporal, a quienes no se ha despedido con ocasión de la crisis desencadenada a partir del año 2008, a pesar de que esto ha obligado a la empresa a reducir los salarios del conjunto de los trabajadores. Este hecho pone de manifiesto el grado de solidaridad alcanzado en el seno de la cooperativa por parte de todos sus miembros.

El liderazgo transformador y compartido adoptado por la dirección ha sido el elemento desencadenante del proceso de cambio. El contacto directo de los directivos-líderes con la totalidad de los miembros de la organización ha traído consigo una clara comunicación de la visión de conjunto de la empresa a los trabajadores, $y$ ha actuado como palanca de los cambios efectuados.

\subsection{Organización basada en procesos}

Se produce un cambio sustancial en la organización del trabajo al introducirse los ELC (Equipos de Línea-Cliente). Estos equipos son el eje a través del cual se proporciona un mejor servicio al cliente, con una mayor participación de los trabajadores. Se trabaja teniendo como centro al cliente y la organización se pone su servicio. La introducción de la reingeniería es una fuente de innovación de los procesos de trabajo, y hace que se aborden cuestiones que van más allá de la mejora continua.

Este tipo de planteamiento basado en los procesos exige pasar de gestionar actividades y tareas a gestionar procesos completos y complejos. La implantación de la gestión por procesos fue un objetivo primordial para los promotores del cambio y muchas de las acciones de reingeniería fueron encaminadas hacia la organización de procesos y su rediseño.

\subsection{Contenido de trabajo}

Los trabajadores, a partir del compromiso adoptado en la reunión de planificación semanal, gozan de autonomía para organizar su trabajo, si bien deben coordinarse con otras personas y equipos; de lo contrario, todos saldrían perjudicados. Se trabaja desde la autogestión, no se ficha, y si bien este hecho puede 
resultar anecdótico, no deja de ser una prueba del grado de responsabilidad y compromiso de los trabajadores con la cooperativa y con su futuro.

Se ha generado un cambio en el ejercicio del control que en muchos casos es fuente de tensión y de enfrentamiento en el seno de las organizaciones. El control ha sido sustituido por una mejor coordinación, que los propios trabajadores llevan a cabo. De este modo se hacen innecesarias figuras intermedias como la de los encargados. A los trabajadores eventuales se les somete a una valoración cada seis meses respecto al desempeño de su trabajo. Esta valoración es realizada por los compañeros que forman parte de su equipo y de ella depende que su contrato sea renovado o no.

Se percibe que el contenido del trabajo ha sido enriquecido de una manera importante dado el grado de autonomía, la capacidad de tomar decisiones existente y la ampliación de contenidos, pero también se percibe un nivel de mayor exigencia y de presión en el desempeño del trabajo dentro de los equipos.

A su vez, se prescinde de la figura del especialista, para evitar que la organización dependa en exceso de determinados individuos que utilicen el conocimiento que han acumulado con el tiempo en beneficio propio, y no en beneficio de la organización. Para ello, se procura que las cuestiones clave para la organización, como pueden ser las relaciones y el trato con los clientes, recaigan sobre muchas personas.

\subsection{Estructura de la organización}

La organización resultante de la aplicación del modelo de gestión es más horizontal, debido a que muchos de los puestos indirectos (encargados etc.) han desaparecido de la estructura. Ello contribuye a una organización más ágil y rápida en la resolución de problemas, dado que las relaciones son más directas al desaparecer las intermediaciones innecesarias. Se ha tratado de romper con la organización jerarquizada, y esta se ha sustituido por una organización más horizontal.

Estos cambios en la estructura han traído consigo la reducción en el número de niveles y la creación de unidades organizativas más autónomas y responsables de las actividades realizadas. Además, ha conllevado la reducción del organigrama, enfocada hacia un modelo horizontal, mediante la cual se han descentralizado actividades y servicios cuya base son los procesos sujetos a reingeniería.

\subsection{Participación de los trabajadores}

La participación de los trabajadores en el proceso de implantación del nuevo modelo de gestión desde el inicio a la actualidad ha sido muy intensa. Fueron los 
trabajadores de AMPO quienes decidieron por abrumadora mayoría iniciar el proceso de cambio y son ellos quienes, a través de su participación en los equipos, están protagonizando el proceso de cambio. Sin esta participación, el modelo no podría tener continuidad, de manera que el reto de la organización es mantener la participación y acrecentarla en todos los sentidos. La participación en los equipos se realiza de manera continua y es la manera natural de proceder con la implantación del nuevo modelo de gestión.

\subsection{Gestión de las personas}

La gestión de las personas está encaminada al logro de una mayor implicación y participación en el proyecto. Se ha impulsado la formación de los trabajadores en la asunción de nuevas competencias y habilidades para el mejor desempeño de su trabajo. El objetivo es lograr trabajadores polivalentes capaces de asumir diferentes tareas y funciones; una de las más relevantes es la capacidad para liderar equipos. Para ello, la formación resulta imprescindible.

\subsection{Cultura organizativa}

El proceso de cambio organizativo ha ido calando progresivamente en el cambio de la cultura organizativa. No es posible que el cambio perdure en el tiempo si no se va consolidando un cambio en los valores, los sentimientos y las actitudes de los trabajadores que conforman la organización. Para ello es necesario perseverar en el tiempo y mantener unas políticas coherentes de manera prolongada. En AMPO, después del tiempo transcurrido desde el inicio del proceso, se percibe un cambio cultural que se va consolidando y que se hace evidente en el cambio de comportamientos y de rutinas. Valores como la identificación con la cooperativa y la orientación al cliente han ido calando en los integrantes de AMPO.

Estos comportamientos, no obstante, están también sujetos al momento económico que vive la empresa; cuando la situación económica mejora, los comportamientos y actitudes de los trabajadores se relajan, mientras que cuando la situación empeora, el compromiso y la implicación aumentan. No obstante, la identificación de los trabajadores y la confianza en el nuevo modelo de gestión son muy amplias.

\section{Conclusión}

La aplicación del modelo de gestión a partir del año 2003 en AMPO ha tenido resultados muy positivos desde un punto de vista tanto cualitativo como cuantitativo. 
El incremento de trabajadores en el periodo 2002-2008 ha sido del $37 \%$; el mayor incremento (12\%) se produce en el año 2003, coincidiendo con la implantación del nuevo modelo de gestión. Este hecho es muy significativo, dado que la implantación de la reingeniería ha desencadenado, en general, grandes reducciones de plantilla.

En el mismo periodo, las ventas se incrementaron de una manera espectacular (se multiplicaron casi por cuatro) y los resultados económicos se multiplicaron por siete. Estos resultados de carácter cuantitativo son la consecuencia de los cambios cualitativos que se han producido en AMPO: cambios en el liderazgo y en el estilo de dirección; en la participación y en la gestión de las personas; en los procesos; en la estructura y en el contenido del trabajo, y, por último, en la cultura organizativa. Estos cambios han posibilitado los buenos resultados cuantitativos, junto con una estrategia centrada en el cliente como elemento esencial de todo el proceso.

Los buenos resultados económicos que acompañaron a la implantación del modelo de gestión en un periodo muy limitado (un año escaso) proporcionaron una gran confianza para profundizar en los cambios organizativos. Además, los resultados económicos obtenidos permitieron un reparto de excedentes entre los socios, lo cual provocó un grado de satisfacción muy elevado por el rumbo que se estaba tomando, que, por otro lado, suponía un elevado nivel de exigencia para con el trabajo realizado en el día a día. Todo ello posibilitó un incremento de confianza en el liderazgo y en el modelo de gestión implantados, de manera que se generó un círculo virtuoso de confianza, afianzamiento y profundización en el cambio iniciado.

Hay que señalar, por último, que el proceso de implantar el modelo de gestión cuenta con un marco institucional que favorece y facilita dicha implantación. El marco institucional no es otro que el cooperativo, que, tanto desde el punto de vista normativo como cultural, tiene como eje central el protagonismo de las personas en su proyecto socioeconómico.

\section{Referencias bibliográficas}

Barandiaran Contreras, M. (2000). Ampo Poyam, Elkarlanean, un recorrido en cooperativa. Litografía Danona, Oiartzun.

BAss, B.M. (1985). Leadership and performance beyond expectations. Free Press, New York.

Granovetter, M. (1985). «Economic action and social structureः the problem of embeddedness». American Journal of Sociology, vol. 91, n 3. 
Hammer, M. y Champy, J. (1994). Reingeniería de la empresa. Parramón, Barcelona.

MacGregor Burns, J. (1978). Leadership. Harper \& Row, Publishers, New York.

March, J.G. y Olsen J.P. (1976). Ambiguity and choice in organizations. Universitetsforlaget, Bergen.

Meyer, J.y Rowan, B. (1977). «Institutionalized organizations: formal structure as myth and ceremony». American Journal of Sociology.

Power, W. W. y DiMaggio, P. (eds.). (1991). The new institutionalism in organization analysis. The University of Chicago, Chicago

Putnam, R. (1993). Making Democracy Work: Civic Traditions in Modern Italy. Princeton University Press, Princenton.

Saratxaga, K. (2007). Un nuevo estilo de relaciones para el cambio organizacional pendiente. Prentice Hall, Madrid.

Sсотт, W.R. (1995). Institutions and organizations. Foundations for organizational science. Sage publications, Beverly Hills.

Urteaga, E. (2013). «La teoría de Robert Putnam: originalidad y carencias», Reflexión política, vol. 15, n²9, pp. 44-60. 